\section{ARTIGO ORIGINAL} ORIGINAL ARTICLE

\title{
Análise da efetividade das políticas de restrição social para COVID-19 no município de Fortaleza-CE
}

\author{
Analysis of the effectiveness of social restriction \\ policies for COVID-19 in the city of Fortaleza-CE \\ Luiz Alexandre Moreira Barros'1, Raphael Douglas de Freitas Lucena², \\ Georgia de Melo Castro Gondim³
}

DOI: $10.21115 / J B E S . v 13 . n 2 . p 160-5$

\section{Palavras-chave}

Infecções por coronavírus, políticas públicas, isolamento social

\section{Keywords}

coronavirus infections, publics policies, social isolation

\section{RESUMO}

Objetivo: Diante da problemática da COVID-19, o município de Fortaleza - CE se destaca pela alta taxa de letalidade verificada até o momento. Para inibir a propagação da doença, os gestores implementaram medidas restritivas para a sua contenção, para, com isso, diminuir as chances de sobrecarregar os leitos hospitalares disponíveis para o tratamento. Nesse sentido, o presente artigo tem como objetivo analisar a efetividade das políticas de isolamento social no município de Fortaleza-CE. Métodos: Para tanto, foi utilizado, como suporte empírico, o modelo SIR (Susceptible, Infectious, or Recovered) proposto por Kermack e McKendrick (1927), para doenças de infecção primária. Os dados para o estudo foram coletados em Cota (2020), no período de 16/03/2020 a 31/05/2020. Resultados: Os resultados mostram que, com o início do isolamento social, a taxa média de transmissão passou de 0,4427 para 0,0945, uma redução de cerca de -75,59\%. Após a implementação do lockdown, a taxa média foi alterada para 0,0349, o que representa uma redução de -88,69\% na taxa de transmissão do vírus. Conclusão: Concluiu-se, portanto, que as implementações de políticas restritivas reduziram a taxa de transmissão e o número de casos da doença.

\begin{abstract}
Objective: Due to the problem of COVID-19, the city of Fortaleza-CE stands out for the high lethality rate observed so far. In order to inhibit the spread of the disease, managers had to implement restrictive measures to contain it, and thereby reduce the chances of overloading the hospital beds available for treatment. In this regard, this article aims to analyze the effectiveness of social isolation policies in the city of Fortaleza-CE. Methods: For this, the SIR model (Susceptible, Infectious, or Recovered) proposed by Kermack and McKendrick (1927) was used as empirical support for diseases of primary infection. The data for the study were collected in Cota (2020), between the period from 16/03/2020 to 05/31/2020. Results: The results show that, with the beginning of the quarantine, the average transmission rate went from 0.4427 to 0.0945 , a reduction of about $-75.59 \%$. After the implementation of the lockdown, the average rate was 0.0349 , which represents a $-88.69 \%$ reduction in the virus transmission rate. Conclusion: It is concluded, therefore, that the implementation of restrictive policies reduced the transmission rate and the number of cases of the disease.
\end{abstract}

Recebido em: 06/06/2020. Aprovado para publicação em: 17/10/2020.

1. Mestrando em Economia no Centro de Aperfeiçoamento de Economistas do Nordeste da Universidade Federal do Ceará (CAEN/UFC), Fortaleza, CE, Brasil.

2. Doutorando em Economia no Centro de Aperfeiçoamento de Economistas do Nordeste da Universidade Federal do Ceará (CAEN/UFC), Fortaleza, CE, Brasil.

3. Mestranda do Programa de Pós-Graduação em Fisioterapia e Funcionalidade da Universidade Federal do Ceará, Fortaleza, CE, Brasil.

Instituição onde o trabalho foi executado: Universidade Federal do Ceará.

Financiamento: Não houve financiamento para a realização da pesquisa.

Apresentação em congresso: Não houve apresentação do trabalho em congresso.

Autor correspondente: Luiz Alexandre Moreira Barros. Centro de Aperfeiçoamento de Economistas do Nordeste da Universidade Federal do Ceará (CAEN/UFC). Av. da Universidade, 2.762, Benfica, Fortaleza, CE, Brasil. CEP: 60020-181.

E-mail: luizalexandremoreira21@outlook.com 


\section{Introdução}

O novo coronavírus, o vírus da síndrome respiratória aguda grave (SARS-CoV-2, em inglês), apresenta diferentes quadros clínicos e, consequentemente, desfechos variáveis, que perpassam desde a afecção assintomática até o óbito por disfunção orgânica múltipla. Entre esses casos, as condições assintomáticas e sintomáticas leves representam a maioria dos indivíduos infectados, porém 15\% dos casos podem revelar necessidade de internação em unidades de terapia intensiva (UTIS). O desenvolvimento dessa enfermidade foi denominado de COVID-19 pela Organização Mundial de Saúde (OMS) (Nascimento et al., 2020; Caetano et al., 2020).

Devido ao alto nível de contágio e mortalidade por essa doença, em escala global, a OMS declarou, em janeiro de 2020, que a COVID-19 seria Emergência de Saúde Pública de Importância Internacional (ESPII), e, em março, atingiu a classificação de pandemia (Caetano et al., 2020).

De fato, o contágio por COVID-19 levou a óbito mais de 350.000 pessoas em todo o mundo e houve, pelo menos, 30.000 mortes no Brasil, até o momento. Dentre os municípios brasileiros, Fortaleza-CE se destaca pelo alto nível de letalidade da doença - o número de óbitos já ultrapassa 2.000 - e pelo número de casos confirmados, que somam mais de 24.000 até o momento. Em consequência disso, os agentes governamentais lançaram estratégias para conter a propagação do vírus, utilizando-se, para isso, da reestruturação dos espaços sociais e das diversas atividades econômicas.

Uma das estratégias mais utilizadas pelos países com grande número de casos da doença foi a política de restrição social e o lockdown. No estado do Ceará, isso se concretizou por meio do Decreto n 33.519, de 19/03/2020, que visa evitar a contaminação e a propagação da doença por meio do isolamento social e da quarentena. Ficou definida, portanto, a suspensão de diversas atividades econômicas, como as industriais (com exceção das essenciais), e as de transporte rodoviário intermunicipal e metropolitano de passageiros, e essas entraram em vigor a partir do dia 23/03/2020. Além disso, houve também o Decreto municipal no 14.663, de 05/05/2020, em Fortaleza, que dispôs sobre medidas de contenção mais rígidas de isolamento social, que entrou em vigência no dia 08/05/2020.

Ocorre, entretanto, que os efeitos dessa reestruturação social são responsáveis por afetar o lado real da economia, levando a uma redução da atividade econômica. Com isso, percebe-se a importância de equilibrar os danos econômicos causados pela COVID-19 e a necessidade de reduzir a transmissão viral, de forma que haja uma política de isolamento mais adequada para a realidade de cada município brasileiro, como em Fortaleza-CE.

Nesse sentido, o presente estudo tem como objetivo analisar a efetividade das políticas de isolamento social (restrição do comércio e lockdown) no município de Fortaleza-CE, durante a pandemia da COVID-19. Para tanto, essa abordagem seguirá a estratégia empírica proposta por Kermack \& McKendrick (1927) para infecções primárias, como evidenciado por Bao et al. (2020) para COVID-19.

\section{Métodos}

Com o objetivo de analisar a efetividade das políticas de isolamento social (restrição do comércio e lockdown) no município de Fortaleza-CE, optou-se por dividir as ações em dois momentos. No primeiro, são calculadas as taxas de velocidade transmissão da COVID-19 ( $\beta$ ) desde o começo da série, no dia 15 de março de 2020, até o dia 30 de maio de 2020; com isso, obtendo-se taxas de transmissão diária. No segundo, será utilizado o modelo SIR (Susceptible, Infectious, or Recovered), largamente utilizado na simulação epidêmica em uma determinada população. Por meio da utilização desse modelo, será possível obter os cenários de transmissão da doença, após a aplicação das medidas de contenção social.

\section{Modelo SIR}

O modelo SIR, proposto por Kermack \& McKendrick (1927), descreve um cenário no qual o sujeito pode passar por três estágios durante o período de contágio: o indivíduo inicialmente é suscetível à doença, depois se infecta com a doença e, por fim, pode se recuperar ou falecer. Define-se o sistema de equações diferenciais do modelo SIR, sendo S a população suscetível à COVID-19, I a parte da população infectada, $\mathrm{R}$ a população recuperada, $\mathrm{N}$ o tamanho da população, $\beta$ a taxa de transmissão da COVID-19 e $\gamma$ a taxa de recuperação:

$$
\begin{gathered}
\frac{d S}{d t}=\beta \frac{S I}{N} \\
\frac{d R}{d t}=\gamma I \\
\frac{d I}{d t}=\beta \frac{S I}{N}-\gamma I
\end{gathered}
$$

A equação (1) mostra a quantidade de pessoas suscetíveis a se infectar com COVID-19 ao longo do tempo, uma equação simétrica e que evidencia o número de casos acumulados de infecções. Já a equação (2) apresenta a quantidade de pessoas que se recuperam da doença ao longo do tempo, sendo calculada por meio da taxa de média de recuperação (ү) vezes o número de indivíduos infectados.

Além disso, a equação (3) mostra a dinâmica do número de casos de COVID-19 ao longo do tempo em uma população. A equação (3) é composta pelo módulo da equação (1), que é o número total de casos de pessoas infectadas por COVID-19, subtraído da equação (2), que apresenta o número de pessoas infectadas. Então, define-se a taxa de casos totais da doença como:

$$
\frac{d I_{t}}{d t}=\beta \frac{S I}{N}
$$

Na equação (5), a variável $\beta$ indica a velocidade de crescimento e a magnitude no número de novos casos totais da doença na população. Assim, um valor alto para $\beta$ indicaria 
uma alta velocidade no número de casos e um grande número de casos registrados de COVID-19, e um valor baixo para $\beta$ apresentaria o efeito contrário. Como apresenta Kirkeby et al. (2017), o $\beta$ pode ser calculado da seguinte forma, sendo $I_{N}$ o número de casos diários:

$$
\beta=\frac{-\ln \left(1-I_{n} / I\right)}{T S / N}
$$

Ademais, para encontrar o número acumulado de casos de COVID-19, é utilizada a seguinte equação, que se configura como uma função cumulativa da taxa de crescimento do número de casos:

$$
\int_{t_{0}}^{t_{\mathrm{n}}} \frac{d I_{t}}{d t} d t=I_{T}
$$

Para verificar a eficiência da simulação, o presente trabaIho utilizou o teste t de Student para ambos os grupos (simulado e real), de forma a identificar se eles são estatisticamente diferentes ou não. Para testar o desempenho da simulação nos períodos de pré-intervenção, foram utilizadas as seguintes hipóteses, sendo $I_{m}^{p r}$ a média dos números de casos do modelo simulados e $I_{r}^{p r}$ a média dos dados reais, ambos no período de pré-intervenção:

$$
\begin{aligned}
& H_{0}: I_{m}^{p r}=I_{r}^{p r} \\
& H_{0}: I_{m}^{p o} \neq I_{r}^{p o}
\end{aligned}
$$

Para verificar o ajuste do modelo aos resultados reais, não se deve rejeitar a hipótese nula, para que os dados possam ser estatisticamente iguais.

Para testar o desempenho da simulação nos períodos pós-intervenção, também foram utilizadas, por meio do teste t de Student, as seguintes hipóteses, sendo $I_{m}^{p o}$ a média dos números de casos do modelo simulados e $I_{r}^{p o}$ a média dos números de casos reais, ambos no períodos pós-intervenção:

$$
\begin{aligned}
& H_{0}: I_{m}^{p o}=I_{r}^{p o} \\
& H_{0}: I_{m}^{p o} \neq I_{r}^{p o}
\end{aligned}
$$

Para aferir se houve impacto da política de restrição, deve ocorrer uma rejeição da hipótese nula.

\section{Coleta de dados}

Foi necessário estimar as taxas de transmissão e simular modelos SIR, de forma que houve a coleta do número de casos diários e do tamanho da população da cidade de Fortaleza-CE.

Para o número de casos diários, os dados foram obtidos por meio dos boletins epidemiológicos diários, disponíveis nas Secretária de Saúde do Estado do Ceará (SES-CE) e na plataforma oficial do Ministério da Saúde durante o período de 16/03/2020 a 31/05/2020, assim organizados e disponibilizados por Cota (2020). O número da população é uma previsão fornecida pelo Instituto de Geografia e Estatística (IBGE) para o ano de 2019.

\section{Resultados}

Nesta seção, apresenta-se um conjunto de informações a respeito do impacto da política de restrição social no número de casos de COVID-19 no município de Fortaleza-CE. Além de fornecer um panorama a respeito das taxas de transmissão diárias do vírus, busca-se também verificar aspectos relacionados à dinâmica dos diferentes cenários sem os efeitos das políticas de restrições ao longo do tempo.

A análise da dinâmica das taxas de transmissão, antes e após a quarentena, pode ser vista na Figura 1. É possível notar que, em média, a taxa de transmissão antes da quarentena é de 0,4427, com um desvio-padrão de 0,4040. Com o início da quarentena, a taxa média passou para 0,0945, uma redução

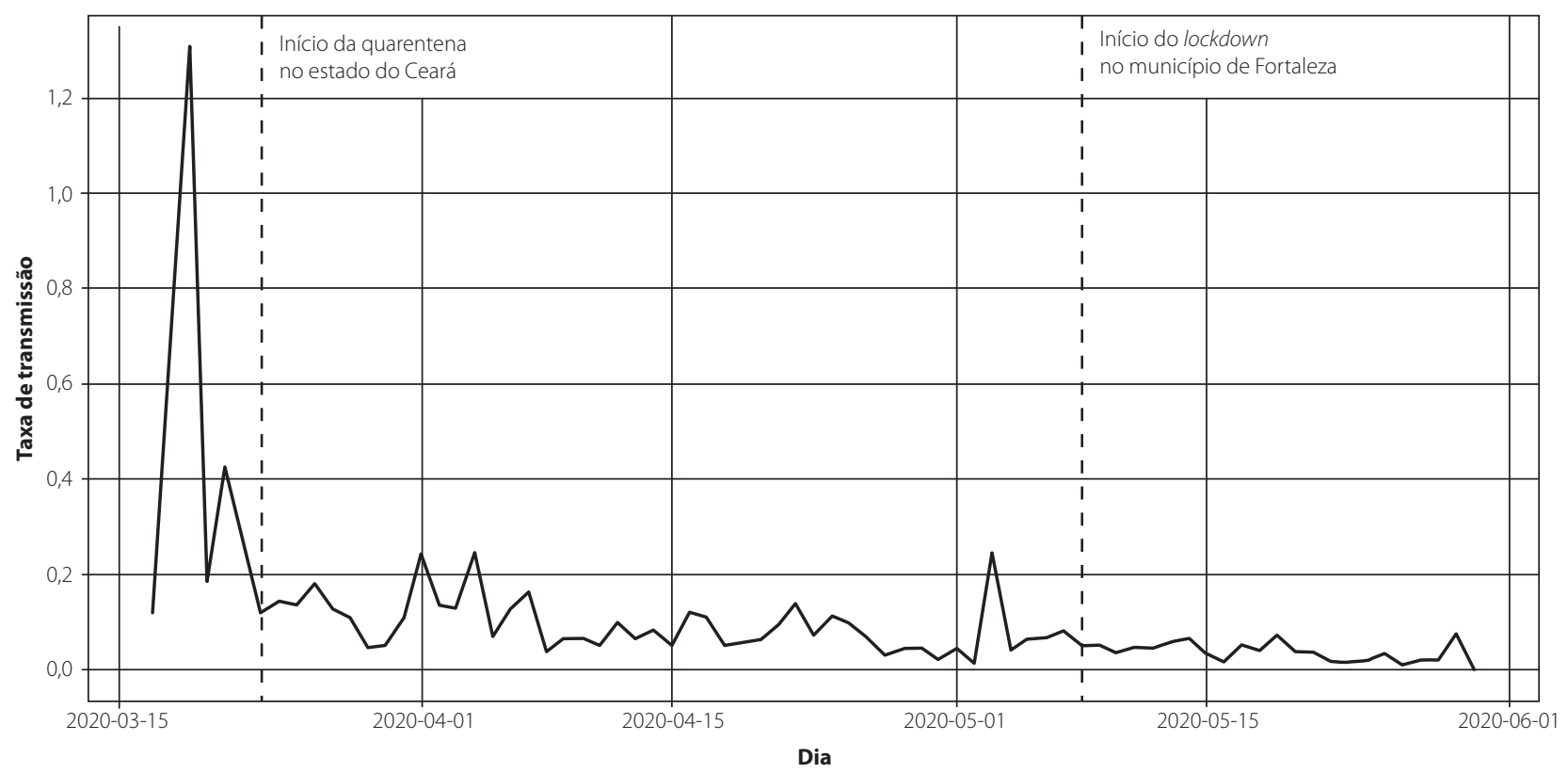

Fonte: Elaboração dos autores, a partir dos dados da pesquisa.

Figura 1. Taxa de transmissão no município de Fortaleza. 
de aproximadamente $-75,59 \%$ nas taxas de transmissão, com um desvio-padrão de 0,0611. Assim, caracteriza-se como uma transição para uma menor volatilidade, quando comparada ao período pré-quarentena.

Após o decreto da política de lockdown, no dia 08/05/2020, foi possível identificar uma maior suavização da volatilidade na taxa de transmissão da COVID-19, juntamente com uma queda mais acentuada do número de casos, com uma taxa média de 0,0349 e um desvio-padrão de 0,0173. Quando comparado ao período pré-lockdown, constatou-se uma redução de -88,69\% na taxa de transmissão do vírus.

$\mathrm{Na}$ Tabela 1, pode-se verificar uma redução da velocidade e uma maior estabilidade das taxas de transmissão diária, após a adoção de políticas de restrição social. Vê-se, durante o período pré-quarentena, que a taxa de transmissão chegou a 1,3099, ou seja, a quantidade de casos em Fortaleza-CE mais do que duplicou durante os dias 19/03/2020 e 20/03/2020. Por outro lado, após a adoção das políticas de restrição, os valores máximos das taxas de transmissão da doença chegaram a 0,263 durante a quarentena e a 0,0804 após o lockdown.

Após o cálculo das taxas de transmissão da COVID-19, foi importante verificar como as curvas de contágio se comportariam sem os efeitos das políticas de restrições. Para isso, foram utilizadas as taxas médias de transmissão $\beta_{\alpha} \beta_{\mu^{\prime}} \beta_{2^{\prime}}$ calculadas na Tabela 1, de forma a utilizar o modelo SIR para prever os diferentes cenários, como é apresentado na Figura 2.
Tabela 1. Estatística descritiva para as taxas de crescimento

\begin{tabular}{|c|c|c|}
\hline Período & Estatística & Valor \\
\hline \multirow[t]{4}{*}{ Pré-quarentena } & Média ( $\beta 0)$ & 0,4427 \\
\hline & Desvio-padrão (ఠ0) & 0,4040 \\
\hline & Máximo & 1,3099 \\
\hline & Mínimo & 0,1177 \\
\hline \multirow[t]{4}{*}{ Quarentena } & Média ( $\beta 1)$ & 0,0945 \\
\hline & Desvio-padrão (01) & 0,0611 \\
\hline & Máximo & 0,2637 \\
\hline & Mínimo & 0,0100 \\
\hline \multirow[t]{4}{*}{ Lockdown } & Média ( $\beta 2)$ & 0,0349 \\
\hline & Desvio-padrão (ఠ2) & 0,0201 \\
\hline & Máximo & 0,0804 \\
\hline & Mínimo & 0,0137 \\
\hline
\end{tabular}

Fonte: Elaboração dos autores, a partir dos dados da pesquisa.

Legenda - $\beta 0, \beta 1$ e $\beta 2$ referem-se às taxas de transmissão sob as condições de cada modelo simulado.

Ao analisar a Figura 2, pode-se inferir que durante o período de pré-intervenção existiu uma ótima sobreposição entre as curvas simuladas e a curva de dados reais. Inicialmente, foi possível notar que, após 20 dias da primeira notificação, a curva simulada com a média das taxas de transmissão pré-quarentena $\left(\beta_{0}\right)$ apresenta uma tendência exponencial,
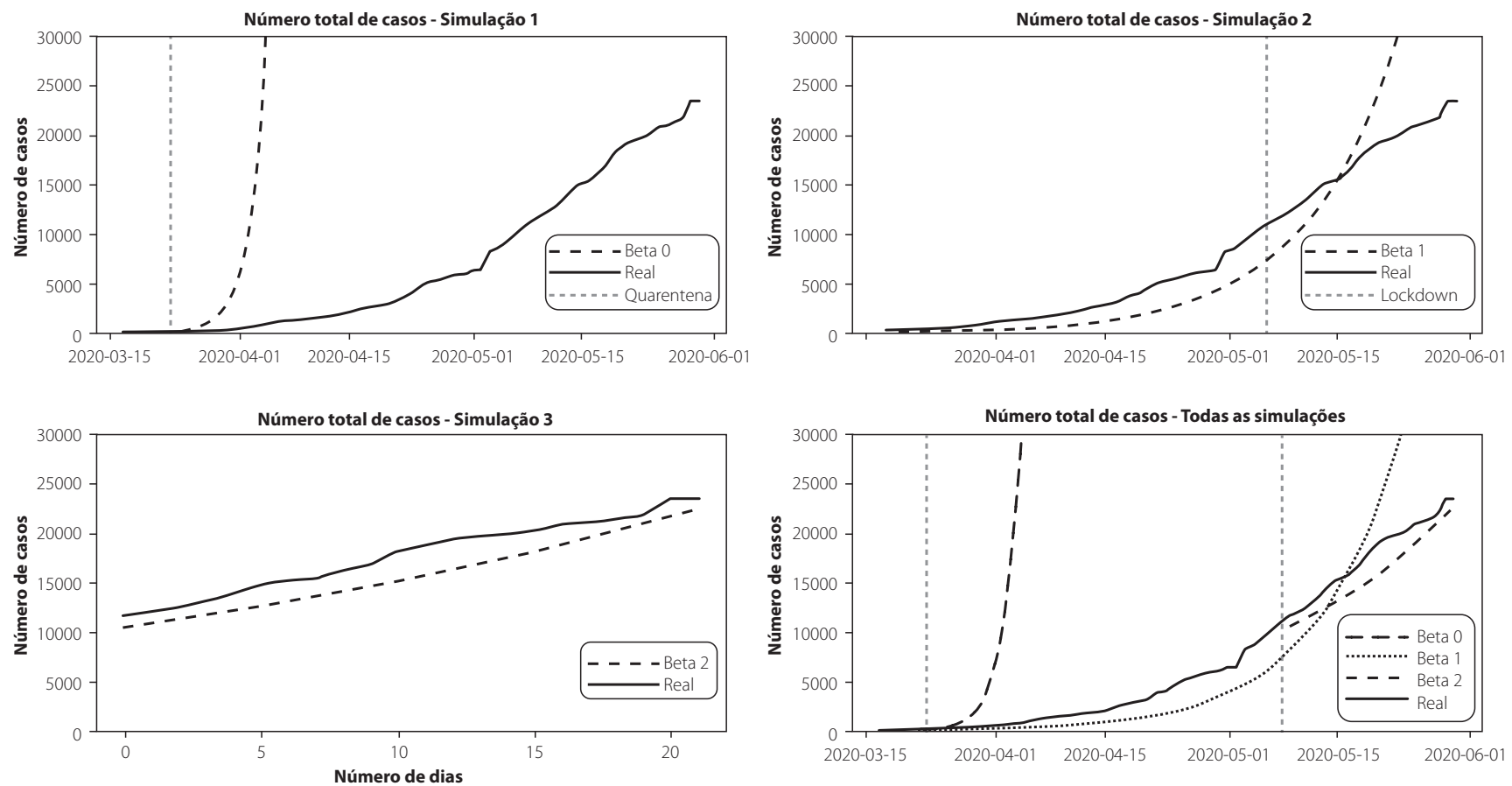

Legenda - $\beta 0$, $\beta 1$ e $\beta 2$ referem-se às taxas de transmissão sob as condições de cada modelo simulado.

Fonte: Elaboração dos autores, a partir dos dados da pesquisa.

Figura 2. Simulações do modelo SIR para Fortaleza. 
prevendo uma total de 2.669.280 números de casos totais para o dia 20/05, enquanto na curva real se observa uma tendência de crescimento, porém com uma menor inclinação.

Com o início do lockdown, após 55 dias da primeira notificação da doença, a curva simulada com a média das taxas de transmissão durante a quarentena $\left(\beta_{2}\right)$ apresentou uma tendência exponencial, o que difere da curva real, gerando um total de 54.853 casos de COVID-19, diferente dos 23.378 casos oficiais registrados para o dia 30/05.

Durante o período do lockdown, obteve-se uma menor variabilidade da taxa de transmissão do vírus em torno da sua média. Na curva simulada com as taxas pós-lockdown, foi possível verificar uma ótima sobreposição da curva real com a curva simulada.

Na Tabela 2, foi apresentado o desempenho das simulações em relação aos períodos de pré-tratamento e pós-tratamento. É possível verificar que existiu um bom ajuste dos dados obtidos na simulação 1, 2 e 3, em relação aos dados reais, antes do início das políticas avaliadas. Após o período de pós-intervenção, nota-se que existe um descolamento entre os dados reais e os dados obtidos nas simulações 1 e 2, o que evidencia que as políticas de restrição social geram efeitos estatisticamente significantes no retardo da evolução do número de casos de COVID-19, no município de Fortaleza$\mathrm{CE}$, durante o período.

\section{Discussão}

O presente estudo analisou a efetividade das políticas de isolamento social no município de Fortaleza-CE, durante a pandemia da COVID-19. Com isso, buscou-se compreender a dinâmica da taxa de transmissão em situações na qual não existem restrições, nas de quarentena e lockdown, identificando a efetividade em cada uma delas. A estratégia empírica seguiu o modelo SIR proposto por Kermack \& McKendrick, em 1927.

Em relação aos resultados simulados nos diferentes cenários, observou-se que, com a redução da flexibilidade, convergindo para uma situação de lockdown, as taxas de transmissão diminuíram ao longo dos períodos, ou seja, o número de casos previstos foi menor, quando comparado aos diferentes cenários pré-lockdown. Esses resultados corroboram os encontrados em Lau et al. (2020), que apresentaram uma redução significativa das taxas de transmissão após a adoção das políticas de restrição em Wuhan.

Desse modo, em uma situação em que os gestores optam por uma estratégia de um curto período de quarentena, Singh \& Adhikari (2020) chamam a atenção para a ineficácia da natureza dessas políticas, pois a taxa de infectados muda de sinal, mas não é o suficiente para evitar a retomada do aumento no número de casos no futuro. Em contrapartida, uma política de longos períodos restritivos pode ser bem-sucedida para diminuir o número de infectados, além de prevenir uma possível volta do aumento da taxa de transmissão. Alvarez et al. (2020) discutem sobre a eficácia do bloqueio, indicando que a elasticidade da taxa de mortalidade em relação ao número de infectados é um determinante essencial da política ideal. A ideia é a de que, à medida que a curva da taxa de mortalidade fosse ficando mais plana, menor seria o tamanho do bloqueio.

Portanto, os resultados sugerem que, na crise causada pela COVID-19, os gestores precisam agir de maneira rápida para implementar estratégias que sejam as mais efetivas para a realidade de sua cidade. A tomada de decisão a respeito do distanciamento social se mostrou relevante para a redução da taxa de transmissão, e a ação de estender essa política para uma situação de lockdown deixou Fortaleza, ceteris paribus, em um estado de natureza mais favorável.

Outro resultado encontrado chama atenção para a importância do controle do tempo e a intensidade das políticas de restrição social, como foi discutido por Toda (2020). O fato de que, na ausência de medidas restritivas, a taxa de transmissão do vírus é potencializada, podendo atingir toda a população em um intervalo de 70 dias. Caso exista uma política de mitigação, os casos podem ser reduzidos para 6,2\% de infectados, em um mesmo intervalo de tempo.

Sendo assim, a ausência de detecção do vírus pode levar a uma avaliação equivocada sobre a real situação, fazendo com que os governos antecipem as medidas de flexibilização e, com isso, contribuam ainda mais para uma retomada do aumento da taxa de contágio da doença, como é discutido em Li et al. (2020).

Como limitações deste estudo, podemos destacar que o baixo número de testagem dificulta o monitoramento dos casos por COVID-19, potencializando os possíveis problemas

Tabela 2. Testes de significância entre os dados simulados e os dados reais

\begin{tabular}{llllc}
\hline & \multicolumn{2}{c}{ Antes da Intervenção } & \multicolumn{2}{c}{ Após a Intervenção } \\
\cline { 2 - 5 } Simulações & Teste-T & P-Valor & Teste-T & P-valor \\
\hline Pré-quarentena & 0,7540 & 0,4662 & $-12,7200$ & 5,780 e-25** \\
\hline Quarentena & $-0,4255$ & 0,6714 & $-5,3640$ & $5,782 \mathrm{e}-6^{* *}$ \\
\hline Lockdown & 1,6801 & 0,1003 & - & - \\
\hline
\end{tabular}

Fonte: Resultados dos autores, a partir dos dados da pesquisa.

Legenda $-{ }^{* *}$ Hipótese nula rejeitada a $5 \%$. 
de subnotificações. O problema da não detecção do vírus pode levar a uma avaliação equivocada sobre a real situação, fazendo com que os governos antecipem as medidas de flexibilização e, com isso, contribuindo ainda mais para uma retomada do aumento da taxa de contágio da doença, como é discutido em Li et al. (2020). Além disso, a presente análise é limitada pela inexistência de cenários contrafactuais, já que as medidas de restrição são observadas em todo o estado do Ceará, impossibilitando a formação de grupos tratados e não tratados pela política.

Ainda, por se tratar de um modelo determinístico, o SIR apresenta algumas limitações operacionais, de modo que outros fatores como o tamanho da população, acesso a saneamento básico, faixa etária da população, renda, são alguns exemplos de variáveis que também podem estar associadas à redução da taxa de transmissão da doença. Porém, a simulação a partir do modelo SIR nos permite replicá-lo para as informações disponíveis no momento da pesquisa, enquanto os modelos do tipo estocástico não nos dariam condições de fornecer resultados prévios sobre as medidas tomadas para o combate ao vírus, em razão da indisponibilidade de dados de controle atualizados para o mesmo período de tempo da doença.

\section{Conclusão}

Observou-se que, à medida que houve uma redução da flexibilidade, convergindo para uma situação de lockdown, as taxas de transmissão reduziram em um montante de -88,69\%. Além disso, constatou-se que o número de casos previstos é menor (redução de 2.669.280 para 23.378 no número de casos totais), quando comparado aos diferentes cenários pré-lockdown, o que evidenciou uma maior efetividade das políticas de isolamento social.
Portanto, as evidências encontradas até o momento sugerem que políticas restritivas, quais sejam a quarentena e o lockdown, foram positivas na redução da taxa de transmissão da COVID-19 no município de Fortaleza.

\section{Referências bibliográficas}

Alvarez FE, Argente D, Lippi F. A simple planning problem for covid-19 lockdown. Cambridge: National Bureau of Economic Research; 2020.

Bao L, Deng W, Gao H, Xiao C, Liu J, Xue J, et al. Reinfection could not occur in SARS-CoV-2 infected rhesus macaques. BioRxiv. 2020.

Caetano R, Silva AB, Guedes ACCM, Paiva CCN, Ribeiro GR, Santos DL, et al. Desafios e oportunidades para telessaúde em tempos da pandemia pela COVID-19: uma reflexão sobre os espaços e iniciativas no contexto brasileiro. Cad Saúde Pública. 2020;36:e00088920.

Cota W. Monitoring the number of COVID-19 cases and deaths in Brazil at municipal and federative units level. SciELOPreprints. 2020.

Kermack WO, McKendrick AG. A contribution to the mathematical theory of epidemics. Proceedings of the Royal Society of London. Series A, Containing papers of a mathematical and physical character. 1927;115(772):700-21.

Kirkeby C, Halasa T, Gussmann M, Tolf N, Graesboll K. Methods for estimating disease transmission rates: Evaluating the precision of Poisson regression and two novel methods. Sci Rep. 2017;7:9496.

Lau H, Khosrawipour V, Kocbach P, Mikolajczyk A, Schubert J, Bania J, et al. The positive impact of lockdown in Wuhan on containing the COVID-19 outbreak in China. J Travel Med. 2020;27(3):taaa037.

Li R, Pei S, Chen B, Song Y, Zhang T, Yang W, et al. Substantial undocumented infection facilitates the rapid dissemination of novel coronavirus (SARSCoV-2). Science. 2020;368(6490):489-93.

Nascimento JHP, Gomes BFO, Carmo Júnior PR, Petriz JLF, Rizk SI, Costa IBSS, et al. COVID-19 e estado de hipercoagulabilidade: uma nova perspectiva terapêutica. Arq Bras Cardiol. 2020;114(5):829-33.

Singh R, Adhikari R. Age-structured impact of social distancing on the COVID-19 epidemic in India. arXiv preprint arXiv:200312055. 2020.

Toda AA. Susceptible-infected-recovered (SIR) dynamics of Covid-19 and economic impact. arXiv preprint arXiv:2003.11221. 2020. 